\title{
Comparison of the risk perception and warning response to air pollution on an individual level in China
}

\author{
Fangping Wang ${ }^{1,2 *}$, and Fei $\mathrm{Su}^{1,2}$ \\ ${ }^{1}$ China Transport Telecommunications \& Information Center, Beijing 100011, People's Republic of China \\ ${ }^{2}$ National Engineering Laboratory of Transportation Safety \& Emergency Informatics, Beijing 100011, People's Republic of China
}

\begin{abstract}
Individual responses to China's heavy air pollution early warning are poorly understood. This knowledge gap has hampered the evaluation and improvement of the early warning system in providing the targeted populations with effective protection guidance. In order to explore the public's response to air pollution warning, field survey were conducted in three major cities of China in 2016. The results indicated that different levels of air pollution warnings were correctly understood in these three cities, but the warning response rate was low. Significant differences in the public's risk perception were demonstrated. Public perception of the health impacts of air pollution (HEP) and knowledge of the warning index (AQI) were significantly higher in Beijing than in Shenzhen. The public perception of the pollution level (DEVIATION) was equal in Beijing and Shenzhen, but higher than that in Shanghai. Gender, education, and risk perception were crucial factors influencing the public's willingness to respond to warnings. Early warning policymakers can use this research to optimize the design and dissemination of early warning information to improve the public's health and quality of life in cities with air pollution.
\end{abstract}

\section{Introduction}

Studies on long-term air pollution exposure have indicated that the chronic effects of air pollution exposure far outweigh its acute effects[1]. Health effect assessments of long-term exposure to air pollution in epidemiological studies[2] have shown that pollution concentrations exponentially increase health hazards, so air pollution warnings are an important way to provide rapid alerts about poor air quality to help the public take protective actions in a timely manner. China is one of the most polluted developing countries in the world, especially in the Beijing-Tianjin-Hebei region, the Yangtze River Delta, and the Pearl River Delta. In response to the frequent occurrence of heavy air pollution on a large scale and the challenges to public health, the Chinese State Council announced its Air Pollution Control Action Plan in 2013, which aimed to decrease the concentration of PM in these three regions by $25 \%, 20 \%$, and $15 \%$, respectively, by 2017[3](CAAC, 2013). Air pollution warnings were a part of this strict target for public health and emissions control.

Current warning issuance in China relies mainly on the objective measures of air quality, with little attention being paid to human perception and responsive behavior [3]. Moreover, air pollution warnings are only reminders, not mandatory instructions, so understanding the public's risk perception and warning response could help elucidate the effectiveness of the warning issuance and establish an effective communication framework to provide better guidance for the public's health protection. The public's response to health risks may mainly be driven by its risk perception of air pollution [4]. Perception varies with demographic characteristics[5] as well as socioeconomic status[6], health status[7], surroundings[8], temperature variations and thermal sensations[9], location effects[10], ways of perception [11](Johnson, 2012), and psychological and lifestyle factors (e.g., time spent in engaging in outdoor activities) [12]. All of these variables can lead to differences in the public's risk perception of air pollution. These factors are not easily measured, so risk perception as a representative factor in an early warning response model must be considered.

Little work has been conducted on discovering the effects of sets of factors (like individual characteristics or risk perception factors) on the air pollution warning response. Merely passing risk information on to the public without telling people what to do to ensure their personal safety is not an effective warning strategy. Understanding how the public responds to the warning is vital to determine what type of protective information should be broadcast to targeted subgroups of the population[13]. An effective early warning system increases the public's trust in warning policy measures and improves residents' personal capacities to mitigate health harms induced by air pollution.

In this study, we examined the public's warning response and factors affecting the warning response by comparing 3 major cities in China. As far as we know, this study is the first attempt to explore the individual

*Corresponding author: wangfangping@cttic.cn 
response to air pollution warnings in urban China, where urban residents are frequently exposed to air pollution. This study investigated (1) the determinants of the perceived local air quality by combining multiple city results, (2) the population differences in the warning response.

\section{Materials and methods}

\subsection{Questionnaire survey}

The aim of the questionnaire survey was to explore the public's willingness to respond to early warnings to different air pollution warning levels. Intense air pollution appears frequently in China, especially in the Beijing-Tianjin-Hebei region, Yangtze River Delta, and Pearl River Delta. Three major cities (Beijing, Shanghai, and Shenzhen) were selected from these regions; the inclusion criteria were the significant differences in the pollution background and warning issuance standards. Beijing is the capital city, Shanghai is an important economic, financial, trading and shipping center, and Shenzhen is the first special economic zone in China; these cities were selected as the representatives of the above regions in our study (shown in Fig. 1).

A stratified random sampling survey was conducted in Beijing, Shanghai, and Shenzhen in 2016, covering
893,741 , and 759 respondents, respectively; a total of 2137 valid questionnaires $(880,583$, and 674, respectively) were returned with a response rate of $89.3 \%$.

The questionnaire mainly consisted of 3 sections. The first section included 3 dimensions of risk perception to measure the individual perceptions of air pollution. The 3 cities' air pollution emergency plans involved air pollution warnings based on the Air Quality Index (AQI) and pollution duration days. Thus, the AQI was utilized as a risk perception factor. The risk perception factors included 3 categories, defined as the DEVIATION (the difference between perceived pollution level and measured pollution level), HEP (respondent's perception of the health effects of air pollution), and AQI (familiar with the Air Quality Index). The response to each question was ranked on a 5-point Likert-type scale ranging from 1 (minimum) to 5 (maximum). The second section investigated the respondents' warning response level. The warning level was designed using the city's air pollution emergency plan. The last section of the questionnaire was designed to collect the respondents' demographic characteristics, including gender, age, education, income, family number (FN), residence time (RT), and individual self-reported health status (HS) on the day of the survey.

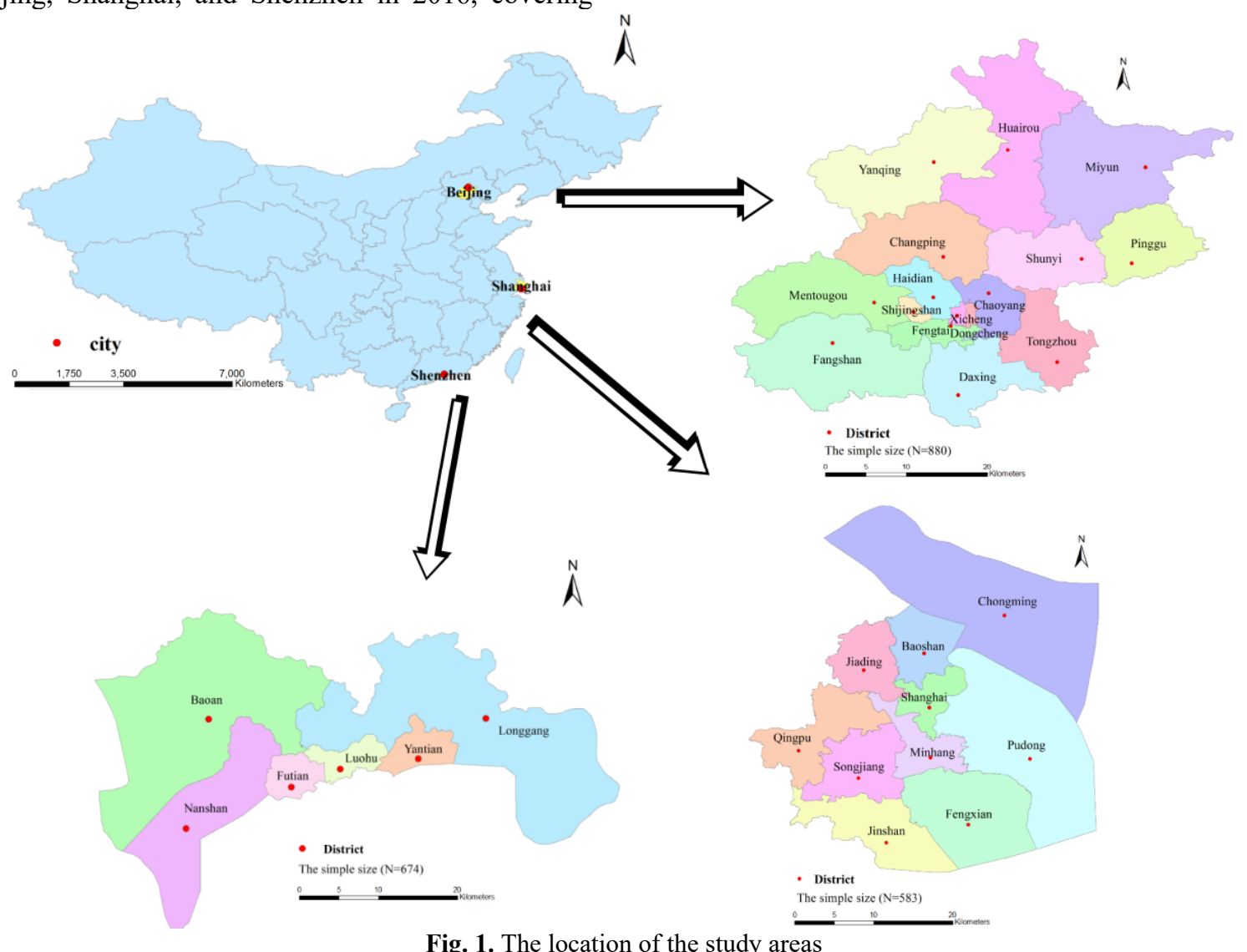

Fig. 1. The location of the study areas

\subsection{Statistical analysis}

The descriptive statistical method was used to present the willingness to respond at different warning levels. The independent-sample t-tests conducted using SPSS 23.0 software were used for the comparison analysis of the risk perception factors in different areas and demographic characteristics. Logistic regression models were employed to examine the factors driving the 
public's warning response. The dependent variables were the warning response. The independent variables included the following: Age was divided into 6 groups: $<20=1,20-29=2,30-39=3,40-49=4,50-60=5$, and $>60=6$; education was divided into 5 groups: compulsory education $=1$, senior high school $=2$, technical qualification $=3$, undergraduate $=4$, and graduate $=5$; income was divided into 5 ranges: $\leq 3000=1$, $3001-6000=2, \quad 6001-9000=3, \quad 9001-12000=4$, and $>12000=5 ; \mathrm{FN}$ was divided into 5 ranges: one person $=1$, two persons $=2$, three persons $=3$, four persons $=4$, five and above $=5$; other binary variables included gender $($ male $=1$, female $=0)$ and $\mathrm{HS}(\mathrm{HS}=1$, non-HS $=0$ ). The last continuous variable included the RT. $P$ values were used to indicate the significance of the effects, and $\mathrm{P}<0.05$ was chosen to indicate statistical significance. All data analysis was implemented in SPSS 23.0 .

\section{Results and Conclusions}

\subsection{Comparison analysis of public risk perception factors}

The mean values of the AQI were 2.03, 1.97, and 1.92 in Beijing, Shanghai, and Shenzhen, respectively. Moreover, the mean values of the HEP were 2.38, 2.07, and 1.86, respectively, while the DEVIATION values were 2.46 , 2.37 , and 2.46 , respectively. In addition, we compared the differences in the public's risk perception among the 3 cities through an independent-sample t-test analysis. It indicated that the AQI in Beijing was significantly higher than in Shenzhen $(t=2.343, p=0.019)$. There was no difference in the AQI values of Beijing and Shanghai.
The value of the HEP in Shanghai was significantly higher $(t=6.372, p=0.000)$ than in Shenzhen, but significantly lower $(t=9.848, p=0.000)$ than in Beijing. However, the DEVIATION values for Beijing and Shenzhen were equal and significantly higher than in Shanghai $(\mathrm{t}=2.204, \mathrm{p}=0.028 ; \mathrm{t}=-2.461, \mathrm{p}=0.014)$.

In conclusion, there exist great differences in the public's risk perception of air pollution in different areas. The public's knowledge of the AQI was similar in Beijing and Shanghai, but the values for HEP and DEVIATION were significantly higher in Beijing than in Shanghai. The values for the AQI and HEP of respondents in Beijing were significantly higher than in Shenzhen. However, people maintained the same perception of the pollution level in Beijing and Shenzhen.

\subsection{Analysis of warning responses}

Considering the sensitivity differences between the populations towards warning signals, it was supposed that subgroups who were willing to take protective measures in response to blue warnings would also respond to the relatively higher warning levels (yellow, orange, and red). Wogalter et al. evaluated the effectiveness of air pollution warnings using behavioral intent[15].

Fig. 2 presents the proportion of the response to different warning levels in the three cities studied. A relatively uniform increase in the proportional response to warnings from blue to red occurs in all three cities. These results indicate that people in these three cities can correctly understand and interpret the different air pollution warning signals; however, the general response to early warnings is not high.

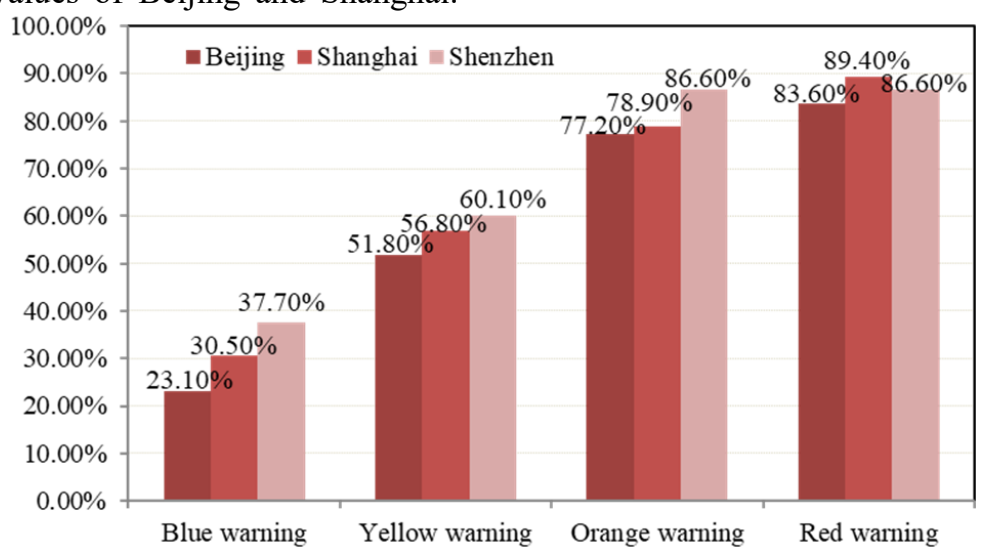

Fig. 2. Distribution of respondents' willingness to respond to air pollution warning signals in Beijing, Shanghai, and Shenzhen

Fig. 3 (a, b, c, d) shows the results of the willingness of the public response.In Beijing, females and those who had higher perceptions of the HEP were more willing to respond to all warning levels. People who knew the AQI preferred to respond from the yellow warning level. Older people were more likely to respond to the blue warning level. In Shanghai, there were various factors that influenced the public's response to the lowest warning level (blue), while only 2 indicators affected the response at the yellow and above warning levels. Those who had higher perceptions of the HEP were more willing to respond to all warning levels. Females were more willing to respond to the orange and red warning levels. In Shenzhen, the more educated people were willing to respond to all warning levels. Females only responded to the highest warning level. People who lived in smaller households were more likely to respond to the lowest warning level. Unlike the people in Beijing, the people in Shenzhen who knew little about the AQI were more willing to respond to the warning signal.

Based on the results of our analysis, the perception that one suffered harm from air pollution could encourage people to respond to the early warnings. However, those who did not realize the health harm of 
air pollution did not respond to the warning signals, which suggests that communication should be enhanced
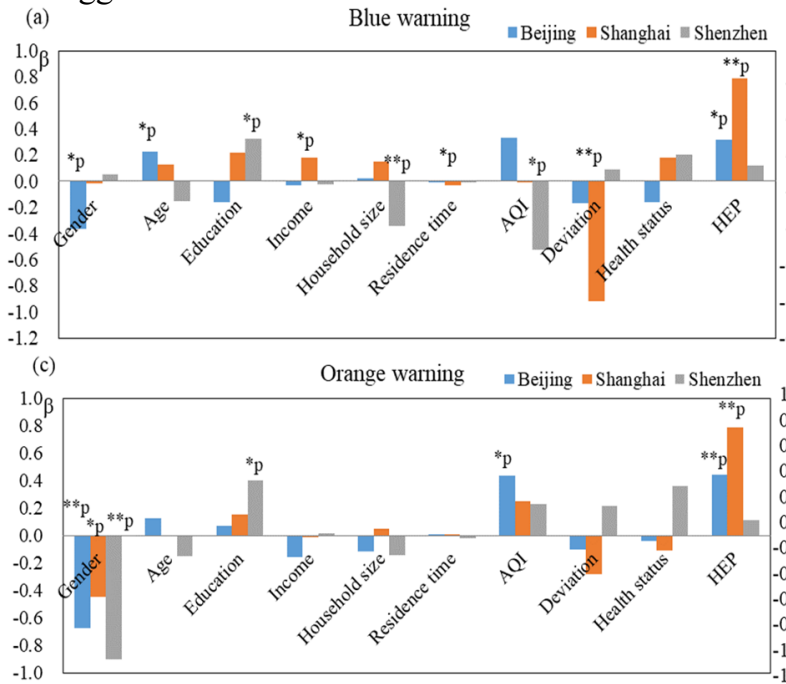

Fig. 3. Regression analysis of the factors influencing the warning

\section{Acknowledgments}

This work was financially supported by the National Key Research and Development Program of China (Grant No.2017YFC0803900).

\section{References}

1. III. C.A. Pope, R.T. Burnett, ,M.J. Thun, E.E. Calle, D. Krewski, K. Ito, et al., Lung cancer, cardiopulmonary mortality, and long-term exposure to fine particulate air pollution, JAMA 28791132 41 (2002)

2. E. Boldo, C. Linares, J. Lumbreras, R. Borge, A. Narros, J. García-Pérez, P. Fernández-Navarro, B..Pérez-Gómez, et al., Health impact assessment of a reduction in ambient $\mathrm{PM}_{2.5}$ levels in Spain, Environ Int 372 342-348 (2011)

3. CAAC, Action Plan on Prevention and Control of Air Pollution. In: S. C. Press, (Ed.), Beijing, China, (2013)

4. S.D. Brody, B.M. Peck, W.E. Highfield, Examining localized patterns of air quality perception in Texas: a spatial and statistical analysis, Risk Anal, 246 1561-1574 (2004)

5. J.C. Semenza, D.J. Wilson, J. Parra, B.D. Bontempo, M. Hart, D.J. Sailor, L.A. George, Public perception and behavior change in relationship to hot weather and air pollution, Environ res, 1073 401-411 (2008)

6. Y.L. Guo, F.F. Liu, Y.A. Lu, Z.F. Mao, H. Lu, Y.Y. Wu, Y.Y. Chu, L.C. Yu, Y.S. Liu, M. Ren, N. Li, X. Chen, H. Xiang, Factors affecting parent's perception on air quality-from the individual to the community level. Int J. Environ. Res Public Health. 135493 (2016).

7. M. Kohlhuber, A. Mielck, S.K. Weiland, G. Bolte, Social inequality in perceived environmental to strengthen the public's awareness about health risks and self-health protection [16].

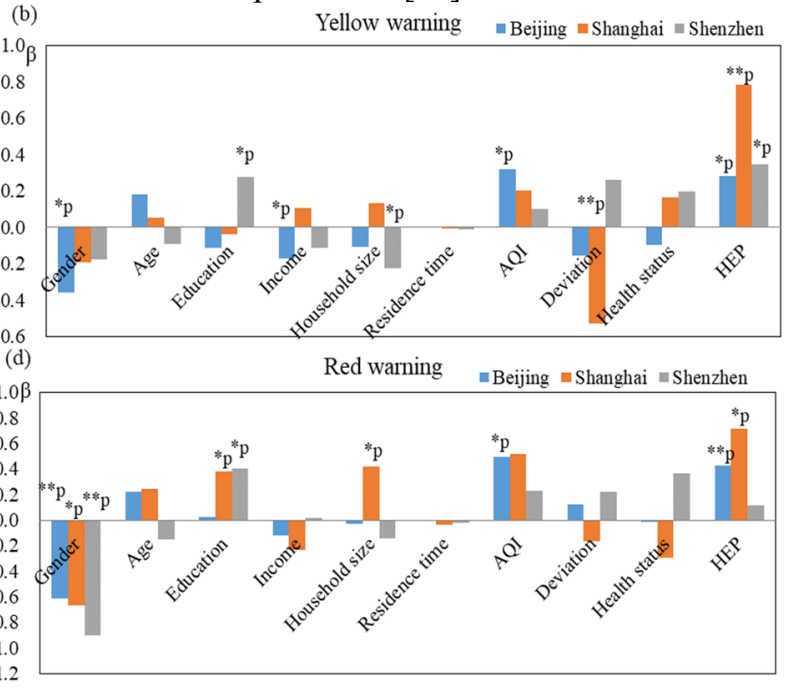

$05, * * p<0.01)$.

exposures in relation to housing conditions in Germany, Environ Res 1012 246-55 (2006)

8. M. Kim, O. Yi, H. Kim, The role of differences in individual and community attributes in perceived air quality, Sci. Total Environ 425 20-26 (2012)

9. H. M. Badland, M. J. Duncan, Perceptions of air pollution during the work-related commute by adults in Queensland, Australia, Atmospheric Environment 4336 5791-5795 (2009)

10. P.V. Dorizas, M-N. Assimakopoulos, M. Santamouris, A holistic approach for the assessment of the indoor environmental quality, student productivity, and energy consumption in primary schools, Environ Monit Assess 1875 1-18 (2015)

11. S.J. Elliott, D.C. Cole, P. Krueger, N. Voorberg, S. Wakefield, The power of perception: health risk attributed to air pollution in an Urban industrial neighbourhood, Risk Anal 19 621-634 (1999)

12. B.B. Johnson, Experience with urban air pollution in Paterson, New Jersey and implications for air pollution communication, Risk Anal 32 1 39-53 (2011)

13. M. Nikolopoulou, J. Kleissl, P.F. Linden, L. Lykoudis, Pedestrians' perception of environmental stimuli through field surveys: focus on particulate pollution, Sci. Total Environ 40913 2493-2502 (2011)

14. R.A. Bradford, J.J. O’Sullivan, I.M. van der Craats, J. Krywkow, P. Rotko, J. Aaltonen, M. Bonaiuto, S. De Dominicis, K. Waylen, K. Schelfaut, Risk perception - issues for flood management in Europe, Nat. Hazards Earth Syst. Sci 12 2299-2309 (2012)

15. M.S. Wogalter, V.C. Conzola, T.L. Smith-Jackson, Research-based guidelines for warning design and evaluation, Appl Ergon 333 219-230 (2002)

16. X. Liu, H. Zhu, Y. Hu, S. Feng, Y. Chu, Y. Wu, C. Wang, Y. Zhang, Z. Yuan, Y. Lu, Public's health risk awareness on urban air pollution in Chinese 
megacities: the cases of Shanghai, Wuhan and Nanchang, Int. J. Environ. Res. Public Health 13 (2016) 